\title{
应用纵向面积分割多次曝光彩虹全息术 合成多幅断层图像的三维单色像"
}

\author{
王明伟 翟宏琛“* 刘福民 母国光
}

(南开大学现代光学研究所, 教育部光电信息技术科学重点实验室, 天津 300071)

\begin{abstract}
摘要采用纵向面积分割多次曝光彩虹全息术对多幅模拟层析图进行全息记录及白 光再现合成三维单色像的实验和理论分析结果. 实验结果及其理论分析表明, 采用纵 向面积分割法将主全息图分割为单元主全息图的记录方法可将多幅断层图像合成为无 视觉灰度及几何畸变的三维单色像. 首次给出了采用该方法合成的一组医学断层图像 的三维单色像.
\end{abstract}

\section{关键词 多次曝光彩虹全息术 全息三维合成 纵向面积分割法 视觉灰度 几何畸变 单色像}

应用多次曝光彩虹全息术, 可把一系列二维的断层平面图像同时记录在全息图上. 使用 白光照明该全息图, 能够将所有的二维断层平面图像同时显示出来, 使观察者不仅能得到每 一断层面的信息, 而且还能同时观察到具有立体空间结构的三维合成图像 ${ }^{[1]}$. 该技术在医学诊 断或工业探伤方面具有不可替代的辅助作用.

传统的记录主全息图的方法是将主全息图的记录区域按横向分割为单元主全息图 ${ }^{[2,3]}$, 用 于分别记录每一张层析图. 此方法存在的问题是, 由于再现白光的色散方向与单元主全息图 的分割方向一致，因而不同景深处的再现像的色彩不同. 由于人眼对各种不同波长的光强主 观感觉不同 ${ }^{[4]}$, 容易造成视觉灰度分布的失真, 影响立体感. 为此需要在记录时采用具有预消 色角的光路安排 ${ }^{[2,3,5]}$ 或采用其他实验方法补偿因色散而引起的几何畸变 ${ }^{[6-8]}$.

本文首次给出的实验结果及其理论分析表明，在多次曝光彩虹全息术中使用将全息图的 记录介质按纵向分割为单元主全息图的方法, 可不借助任何其他复杂的计算或光路安排, 将 各层析图再现为波长一致的单色像, 因而不会产生视觉灰度畸变或几何畸变.

\section{1 记录单元主全息图的横向及纵向面积分割法}

多次曝光二步彩虹全息术的纵向面积分割法是指沿纵向分割主全息图的记录介质, 形成 多个单元主全息图, 如图 1(a)所示. 即 $x-y$ 平面为主全息图 $\mathrm{H}_{1}$ 所在平面, 平行参考光与 $x$ 轴垂 直, 与 $z$ 轴的夹角为 $\theta$. 在记录介质前表面放置一个可沿 $x$ 方向移动的狭缝 $\mathrm{S}$, 狭缝的短边与 $x$ 轴平行, 并与平行参考光 $\mathrm{R}_{1}$ 垂直. 层析图物片紧贴毛玻璃漫散射板 $\mathrm{D}$ 放置且与记录介质平面 平行. 用平行光沿 $z$ 方向照射物片形成漫射物波场. 这样, 在记录多幅断层图片的过程中, 每

* 国家自然科学基金(批准号: 69677020)和天津市自然科学基金(批准号: 99380081)资助项目

**E-mail: zhai@nankai.edu.cn 


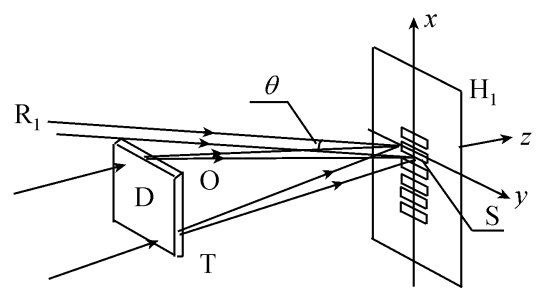

(a)

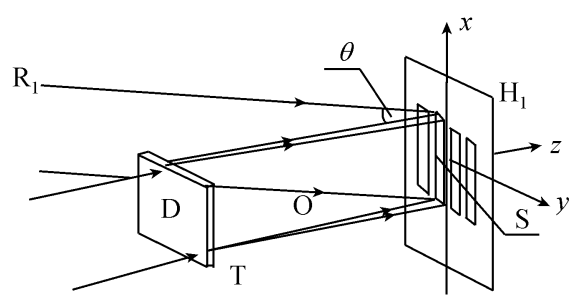

(b)

图 1 记录主全息图的示意图

(a)纵向面积分割, (b)横向面积分割, $\mathrm{H}_{1}$ 示主全息图; $\mathrm{S}$ 示狭缝; $\mathrm{R}_{1}$ 示准直参考光; $\theta$ 示参考光与物光夹角; $\mathrm{D}$ 示散射板；O 示物光； $\mathrm{T}$ 示层析图片

换一幅断层图, 狭缝沿 $x$ 方向移动一个狭缝的距离, 则可得到一个新的单元主全息图. 依此顺 序, 可将按原相对位置摆放的多幅断层图片分别记录在同一主全息图上处于不同狭缝区域内 的单元主全息图上. 在上述分割方法中, 由于单元主全息图的排序取向垂直于参考光和 $z$ 轴所 在的平面, 故称为纵向面积分割法.

在二步彩虹全息术的第 2 步记录过程中, 采用与原记录参考光共轭的再现平行参考光、全 息图 $\mathrm{H}_{1}$, 并仍用平行记录参考光将全部狭缝及其内部单元主全息图的衍射光波记录在第 2 张 全息图 $\mathrm{H}_{2}$ 上. 由于 $\mathrm{H}_{2}$ 位于原物片组的中心图片再现实像面上, 因此第 2 张全息图记录的是原 多幅层析图片再现实像的像面或准像面全息图.

与此对照, 应用传统的横向面积分割法 ${ }^{[2,3]}$ 记录主全息图的光路如图 1(b)所示. 该光路与 图 1(a)不同之处仅在于这里取狭缝的长边与 $x$ 轴平行. 与纵向面积分割法相比, 这相当于将狭 缝的方向旋转了 $90^{\circ}$. 每换 1 张断层图片, 狭缝沿 $y$ 方向移动一个狭缝的距离, 并曝光一次, 则 得到一个新的单元主全息图. 依此顺序可将多幅断层图片分别记录在沿横向分布的不同的单 元主全息图上. 其记录第 2 张全息图 $\mathrm{H}_{2}$ 的过程与采用纵向分割方法的相应记录过程完全一致.

由于在上述两种记录主全息图的光路安排及实验中, 参考光为平行准直光, 物片面积远 小于物片距主全息图的距离, 因此可认为物光场的零频和基频部分主要是沿 $z$ 轴方向传播至 主全息图. 故单元主全息图狭缝区记录的低频干涉条纹的空间取向均近似沿 $x$ 方向. 根据同样 的分析, 在将主全息图再现并记录在第 2 全息图 $\mathrm{H}_{2}$ 上的第 2 步记录过程中, 全息图 $\mathrm{H}_{2}$ 上的干 涉条纹的基频取向亦主要沿 $x$ 方向分布. 其条纹密度与夹角 $\theta$ 有关. 因此, 最后用白光再现应 用了上述任何一种分割方法的彩虹全息图 $\mathrm{H}_{2}$ 时, 均可观察到狭缝组的实像及相应的原断层图 片组的虚像沿 $y$ 和 $z$ 方向色散, 呈彩虹分布.

此与波长有关的色散可由 Champagne 公式 ${ }^{[9]}$ 推得

$$
\begin{gathered}
x_{i}=x_{0}, \\
y_{i}=y_{0}-z_{0} \sin \theta^{\prime}\left[\left(\lambda_{0} / \lambda_{i}\right)-1\right], \\
z_{i}=\left(\lambda_{0} / \lambda_{i}\right) z_{0},
\end{gathered}
$$

其中 $\theta^{\prime}$ 是与最后一步的记录参考光方向共轭的白光再现参考光方向, $\lambda_{0}$ 是记录光波长, $x_{0}, y_{0}$ 及 $z_{0}$ 是若用原记录波长再现全息图时会得到的像点坐标, $\lambda_{i}\left(\lambda_{i}=\lambda_{\mathrm{S}} \cdots \lambda_{0} \cdots \lambda_{1}\right)$ 表示再现白光 中包括的任一波长, $x_{i}, y_{i}$ 及 $z_{i}$ 为与此再现波长对应的再现像点坐标. 


\section{2 白光再现像的不同视觉效果}

若对第 2 步记录的全息图 $\mathrm{H}_{2}$ 进行白光再现，则所有被记录的二维层析图会按其原记录时 所处的空间相对位置被同时再现出来，并能被同时观察到. 此即为全息方法合成的三维再现 像. 但其视觉效果会因记录单元主全息图时分别采用了上述两种不同的分割方法而有所不同.

将在记录主全息图时使用了横向分割方法的全息图进行白光再现时，包括了所有单元主 全息图的再现狭缝实像组会形成一个观察窗口。但由于色散效应, 该窗口会色散为色彩不同 的窗口组, 它们按(1) (3)式沿 $y$ 及 $z$ 方向色散, 如图 2(a)所示. 这样, 当观察者沿某一方向观察 全息图时，其视线会先后通过色彩不同的窗口中记录了不同的层析图的狭缝，同时观察到不 同景深处各层析图的再现像. 则每一幅再现的层析图就会具有相应的不同色彩，即合成的三 维再现像会沿不同景深呈彩虹分布，如图 2(a)所示. 这些呈彩虹分布的再现像除了会产生位置 及尺度上的畸变外(见(1) (3)式), 还会因人眼的视觉对不同波长光的灵敏度不同 ${ }^{[4]}$, 使观察到 的三维再现像与原记录的物片相比, 沿景深方向存在灰度分布的畸变.

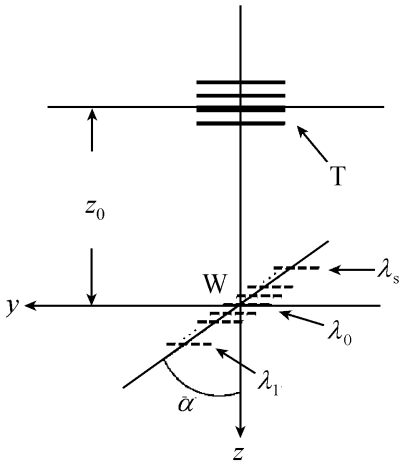

(a)
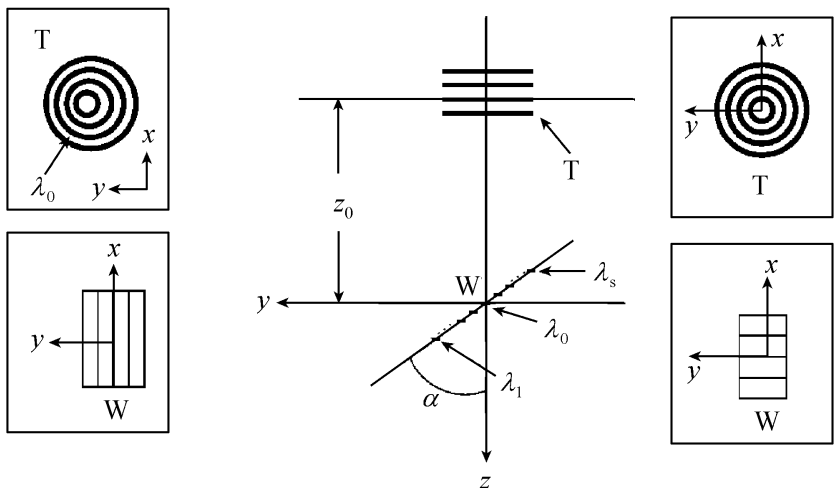

(b)

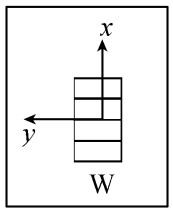

图 2 白光再现的观察窗口

$\mathrm{T}$ 示沿 $-z$ 方向观察到的再现像 (见图 3)； $z_{0}$ 示 $\mathrm{H}_{2}$ 与中心观察窗口的间距； $\alpha$ 示色散角;

$\mathrm{W}$ 示观察窗口; $\lambda$ 示再现波长 $\left(\lambda_{0}\right.$ 示记录波长; $\left.\lambda_{1}>\lambda_{0}>\lambda_{\mathrm{s}}\right)$

而将在记录主全息图时使用了纵向分割方法的全息图进行白光再现时，包括了所有单元 主全息图的再现狭缝实像组也会形成一个观察窗口, 但由于其中的狭缝沿 $x$ 方向依次排列, 即 与色散方向垂直，因而观察者可以在某一视角范围，通过同一窗口中记录了不同的层析图的 狭缝, 同时观察到各层析图的再现像, 如图 2(b)沿 $z$ 轴的观察效果所示. 这样, 所有再现的层 析图就会具有相同的色彩，即合成的三维再现像沿不同景深色彩相同. 因而不会因人眼对不 同波长的光强主观感觉不同而产生灰度畸变. 另一方面, 尽管该组再现像的色彩会随观察角 度的变化而整体改变，但我们总可以找到一个与记录波长 $\lambda_{0}$ 相对应的观察角度，使 $\lambda_{i}=\lambda_{0}$. 根据(1) (3)式可知, $x_{i}=x_{0}, y_{i}=y_{0}$ 及 $z_{i}=z_{0}$. 因而不会存在任何位置、尺度方面的畸变. 总 之，与使用横向面积分割方法相比，使用纵向面积分割方法的实验方便、简单，合成的三维立 体全息图能够更准确地再现原三维物体处于不同景深的断层面的相对灰度、位置及尺度分布, 因而能更真实地再现原三维物体的全貌. 


\section{3 白光再现像的分辨率}

应用面积分割法记录单元主全息图时，其白光再现像的分辨率取决于狭缝像的衍射极限 和色散效应. 对于纵向分割方法, 由(1)式可知，在 $x$ 方向，再现像没有色散，因而白光再现像 在纵向的系统分辨率极限是狭缝的衍射极限，其最小可分辨距离可表示为 ${ }^{[10]}$

$$
E_{x}=\lambda_{i}\left(Z_{0}-Z_{0 i}\right) / w,
$$

其中 $Z_{0}$ 为 $\mathrm{H}_{2}$ 和 $\mathrm{H}_{1}$ 之间的距离, $Z_{0 i}$ 是第 2 次记录时 $\mathrm{H}_{2}$ 和第 $i$ 个断层图像实像的间距, $\lambda_{i}$ 是再 现波长, $w$ 为狭缝宽度. 如果观察者的眼睛位于狭缝实像处, 只要狭缝的宽度 $w$ 大于人眼的 瞳孔尺寸，则 $x$ 方向的衍射不会影响观察时的视觉分辨率.

在 $y$ 方向, 由于观察者的眼睛位于狭缝实像位置, 而且狭缝的长度远大于瞳孔的尺寸, 因 而狭缝的衍射极限对系统分辨率的影响可以忽略, 系统的极限分辨率仅取决于由色散引起的 色模糊. 对于任意一个物点, 其在 $y$ 方向上的色模糊可以表示为 ${ }^{[10]}$

$$
\Delta y=Z_{0 i} l / Z_{0},
$$

这可以看作是 $y$ 方向的系统分辨率极限. 由此式可以看出, 缩短狭缝的长度意味着系统分辨率 的提高. 但是, 狭缝长度的缩短会在实验上降低主全息图再现实像的亮度, 容易引人背景噪声, 使全息图信噪比下降，降低白光再现全息图的衍射效率，从而影响再现像的亮度. 因而，在实 验中要在这两者之间进行权衡. 为此, 可采用重复记录的循环分割记录方法增加记录区域, 即 将主全息图分割成几个依次排列的狭缝组，并在其中重复记录该组断层图像，从而可以通过 增加记录面积来提高主全息图再现实像的亮度. 这样, 既提高了再现像的分辨率, 又保证了再 现像信噪比和亮度. 此外, 采用循环分割的方法还可以增大再现像的视角, 使再现的三维像更 富有层次感.

此外, 从(5)式还可以看出, 分辨率与 $Z_{0 i}$ 直接相关, 因此在第 2 步记录过程中, 将 $\mathrm{H}_{2}$ 置于 从 $\mathrm{H}_{1}$ 再现出的实像的中心位置, 使 $\mathrm{H}_{2}$ 记录的是这些实像的像面全息图或准像面全息图, 也是 提高分辨率的一种有效方法.

\section{4 实验结果}

为了验证并给出使用上述两种分割方法记 录的全息图在白光再现时的不同效果, 我们设 计并完成了一组模拟实验。在实验中，拍摄了 具有不同半径的圆环的一组共 4 张黑白照片, 用以模拟从不同断层获取的一组断层图片(见 图 3), 并分别使用本文介绍的两种方法将其合 成为白光三维立体再现像.

实验中使用波长为 $532 \mathrm{~nm}$ 的 Verdi 激光器 作为实验光源, 各步记录过程中使用的记录介 质均为天津- III 型银盐干版. 在记录过程中, 参 考光和 $z$ 轴夹角保持为 $28^{\circ}$. 记录主全息图时, 模拟层析图组的中心位置到干板的距离为
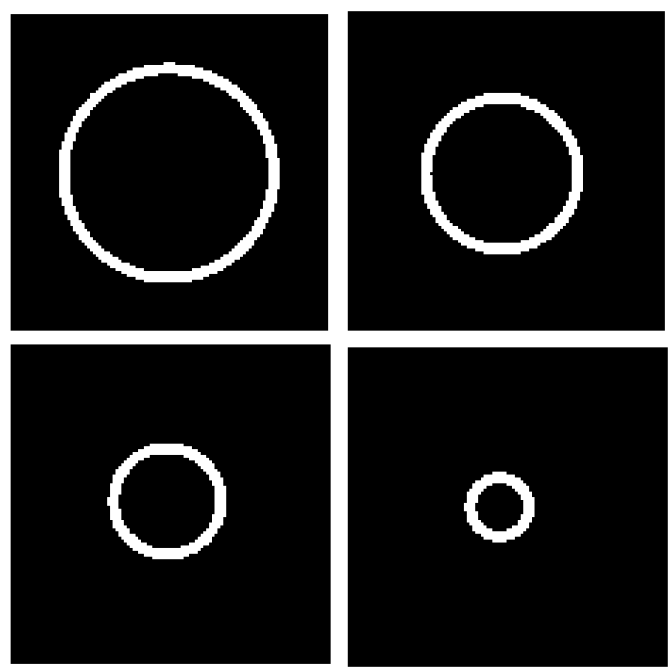

图 34 张半径不同的环形模拟层析图片 

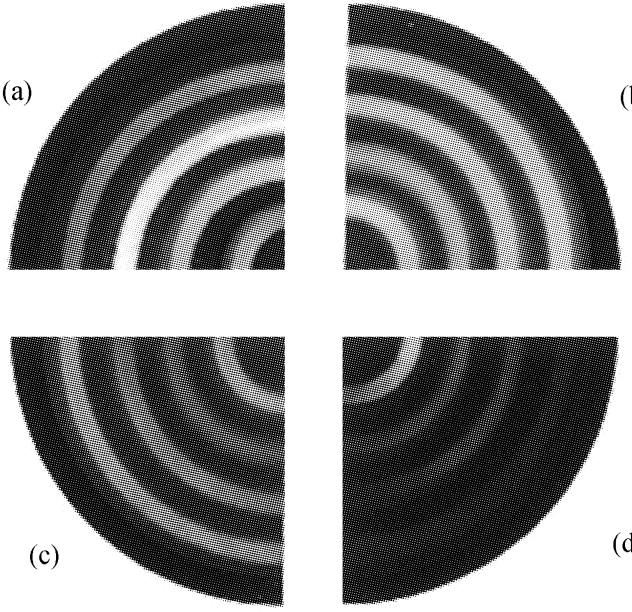

图 4 基于横向面积分割(a)和基于纵向面 积分割 $(b) \sim(d)$ 的全息图的白光再现像 (b) $\lambda=\lambda_{0}$, (c) $\lambda>\lambda_{0}$, (d) $\lambda<\lambda_{0}$
$500 \mathrm{~mm}$. 在横向和纵向分割中, 狭㖓的宽度均 取大于人眼瞳孔的尺寸 $(6 \mathrm{~mm})$, 以消除狭缝的 (b) 衍射极限对人观察时视觉分辨率的影响. 对于 纵向分割, 为了限制色模糊, 在主全息图的记 录过程中, 狭缝的长度限制为 $15 \mathrm{~mm}$. 采用了 上文提到的循环分割方法, 即将主全息图分成 分割成 4 个依次排列的狭缝组, 每张断层图片 分别记录在不同循环区内的相应的狭缝内. 为 进一步提高再现像的分辨率, 实验中将 $\mathrm{H}_{2}$ 置于 从 $\mathrm{H}_{1}$ 再现出的实像的中心位置, 即 $\mathrm{H}_{2}$ 上记录的 均为像面或准像面全息图.

使用横向和纵向面积分割法记录主全息图 的白光再现像分别示于图 4(a) (d). 很明显, 在 采用横向面积分割法记录主全息图的同一组再 现像中, 不同景深上的圆环分别呈现出具有彩 虹分布的不同色彩(图 4(a)); 而在采用纵向面积 分割法记录主全息图的同一组再现像中, 不同景深上的圆环像呈现出相同的色彩, 见图 4(b) (d). 它们是分别从不同的观察角度拍摄到的采用纵向面积分割法的同一张全息图的白光 再现像. 可以看出, 从不同角度上观察同一白光再现像时, 圆环组会由于色散效应, 随观察角 度的变化而呈现出不同的色彩, 但不管观察角度如何变化, 不同景深上的再现像的色彩始终 是保持一致的.

我们还应用本文提出的纵向面积分割法记录了 13 张人头部的层析 MR 片(图 5), 并在白光 再现中首次合成了单色的三维立体图像, 如图 6 所示. 其中, 在脑部中央可以明显地观察到一

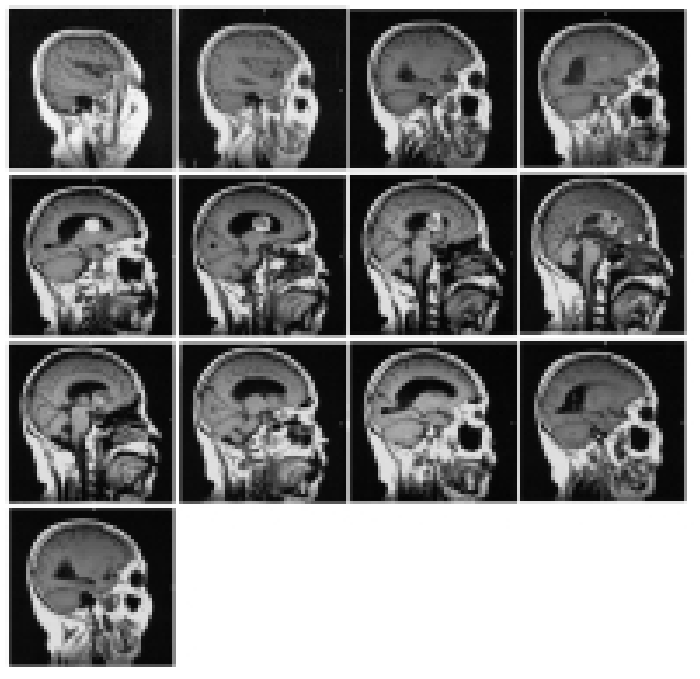

图 513 张人头部层析 MR 图片

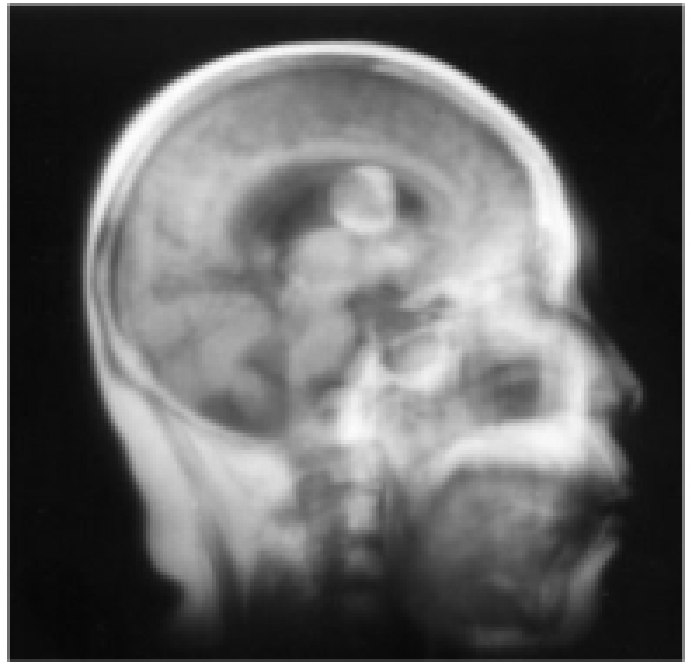

图 6 应用纵向面积分割法合成的 13 张人 头部层析 MR 图片的三维单色再现像 
肿瘤状结构. 该光学三维合成像的分辨率和亮度基本保持了原层析 MR 图片的水平, 能够满足 医疗诊断的各种要求. 由于与原分立的层析 MR 图片相比, 合成的三维像具有直观的立体感, 易于分析各组织结构间的相互位置, 因而该技术的实用化将在医疗诊断中产生不可替代的辅 助作用.

\section{5 结论}

本文首次利用一组专门设计的模拟层析图, 分别采用纵向和横向面积分割方法记录了多 次曝光彩虹全息的主全息图, 实现了该组模拟层析图的三维立体合成. 实验结果及理论分析 表明, 采用纵向面积分割方法与常规的横向面积分割法记录主全息图的技术相比, 其主要优 越之处在于, 无需进行任何的理论计算和补偿措施, 即可在适当的观察角度观察到与记录波 长相同的、不存在任何视觉灰度及尺度畸变的单色三维再现像.

本文还首次给出了应用纵向面积分割方法合成的一组具有病变的人脑层析 MR 图片的三 维单色再现像. 由于此类光学三维合成图像具有明显的直观立体效果, 因而在医疗诊断分析 中将具有不可替代的辅助作用.

\section{参考文 献}

1 Caulfield H J, ed. Handbook of Optical Holography. New York: Academic Press, 1988. 135 136

2 Tsujiuchi J. Medical applications of holographic 3-D display. Goodman J W, ed. International trends in optics. New York: Academic Press Inc, 1991. 297 305

3 Xie J H, Zhao Y L, Yu M W. Traversing area partition technique and its application in 2D/3D embossing holograms. Acta Optica Sinica, 1988, 8(5): 410 416

4 Jacobs G H. Comparative Color Vision. New York: Academic Press Inc, 1981. 189 191

5 Benton S A. Achromatic holographic stereograms (A). J Opt Soc Am, 1981, 71: 1568 1572

6 Guo S G, Zhai H C, Zhan Y L, et al. The distortion and its compensation of the reconstructed images in multiple-exposure rainbow hologram. In: Mu Guoguang, ed. Proc ICO Topical Meeting, Optics for Information Infrastructure, JOEL, 1998, 9 Supp: 214 217

7 Burckhardt C B. Display of holograms in white light. Bell Systems Technical Journal, 1966, 45(4): 1841 1850

8 De Bitteto D J. White light viewing of surface holograms by simple dispersion compensation. Appl Phys Lett, 1966, 9(8): 417 425

9 Champagne E. Nonparaxial image, magnification, and aberration properties in holography. J Opt Soc Am, 1967, 57: 51 55

10 于美文. 光全息学及其应用. 北京: 北京理工大学出版社, 1996. 437 440 